Annals of Warsaw University of Life Sciences - SGGW

Horticulture and Landscape Architecture No 39, 2018: 37-48

(Ann. Warsaw Univ. of Life Sci. - SGGW, Horticult. Landsc. Architect. 39, 2018)

DOI 10.22630/AHLA.2018.39.4

\title{
The historical shrub roses in urban green areas
}

\author{
MARTA JOANNA MONDER
}

Polish Academy of Science Botanical Garden - Center for Biological Diversity Conservation in Powsin

\begin{abstract}
The historical shrub roses in urban green areas. Roses are among the most important ornamental plants as the tradition of their cultivation dates back to ancient times. Nowadays, the historical roses evince our cultural heritage and biodiversity of the genus Rosa. Moreover, the mentioned roses have a high decorative value. The majority of older-origin cultivars meets the conditions required for plants intended for planting in the city, e.g. high winter hardiness, high heat and drought tolerance, sufficient resistance to diseases and pests, moderate or small soil requirements, satisfactory growth on their own roots, and self-cleaning. The low cultivation requirements allow to reduce the costs of maintenance of green areas. The participation of roses in an ecological balance in urban ecosystems is important. There is no tradition of the application of historical roses in public green areas and historical gardens in Poland. However, some of the historical cultivars can be recommended for planting along transport routes and in urban green areas, especially in the public greenery, communal gardens, parks, and in all places of historical importance. Many of them can be used in naturalistic planting schemes for garden landscapes. Roses can be planted solitary, in groups, for mass planting, hedges, and in sunny or partially shaded sites. They are also ideal for compositions with other species.
\end{abstract}

Key words: city landscape, urban gardening, garden roses, old roses, park, historical garden

\section{INTRODUCTION}

Roses are among the most important ornamental plants as the tradition of their cultivation dates back to ancient times. The term Historical Roses refers to the roses known, bred and/or cultivated before the end of World War II. Among historical roses, there are several groups of roses in terms of their origin (Cairns 2003) and, simultaneously, different in terms of their cultivation requirements (Krüssmann 1974), frost tolerance and decorative values (Monder 2007a, b, 2011, 2012a, 2014). Moreover, the historical roses evince our cultural heritage and the biodiversity of the genus Rosa.

Greenery in cities is associated primarily with the street-adjacent areas, and the selection of plants for cities is focused mainly on them (Maranda et al. 2013, Borowski et al. 2016). Nevertheless, the spread of urban development and residential areas entails the need to establish green areas of different character (Baranowski 2010). Nowadays, regardless of the concept and function of the green area, breeders' catalogs predominantly propose numerous universal ground cover roses with different growth characteristics for urban areas, and in recent years also landscape roses and little shrub roses, but more rarely - classic park roses. Normally, a limited number of historical roses is mentioned, although they are valuable in cultivation over the years and often do not have any "substitutes" among modern cultivars (Monder 2012a, b, 2014). In Poland historical roses are seldom cultivated, except from amateur 
gardens, and in this regard they have been growing in popularity for a dozen or so years. Among numerous park roses, there are historic taxa, with features of high utility in urban conditions because of their growth characteristics, e.g. remarkably high winter hardiness, high heat and drought tolerance, sufficient resistance to diseases and pests, moderate or small soil requirements, satisfactory growth on their own roots, self-cleaning and a high decorative value (Monder 2007a, b, 2011, 2012a).

Limited space for trees creates great opportunities for the extensive use of bushes, not just ground cover shrubs. On the other hand, the presence of shrubs makes also a broad improvement of biocenotic conditions for tree survival (Popek 2002, Mędrzycki 2007).

The ways of using park roses are similar to other deciduous shrubs, e.g. groups, gatherings, singles. The single-species (Wizner 1970) or multi-species rose hedges are relatively unpopular, while worthy of being popularized. As the accompanying species, those with different flowering dates or attractive fruits, such as Amelanchier, Prunus, Crataegus, Viburnum, are selected. Usually, these are unformed hedges, but many species tolerate pruning. Such assumptions can fulfill many social, ecological and natural functions.

The paper presents historical shrub roses in terms of their ability to be used in the city, according to the state of the knowledge on them, up-to-date conducted research and observation.

\section{SELECTED ASPECTS OF URBAN ENVIRONMENTAL CONDITIONS AND THEIR CONSEQUENCES FOR ROSES' CULTIVATION}

In the case of roses, especially during the period after the Second World War, nurseries were established around industrial cities such as Łódź. There were no disturbances in the growth of shrubs in the nurseries and the produced budded rootstocks were of good quality (Wizner 1970). Wizner (1970) claims that he also did not observe anomalies in the growth of shrubs planted in the urban environment of the mentioned city. Based on similar practical observations, roses have been recommended for many years to fill the city space in all types of city greenery (Wizner 1970, Krüssmann 1974, Bugała 1991).

Multi-stress conditions prevailing in urban environments, including streets, create unfavorable habitats for small size and ground cover shrubs. Shrubs growing aside roads are directly exposed to pollution from vehicles and road maintenance and they often fade. Especially on roads and sidewalks, the danger areas mainly due to salinity and associated physiological drought. The elevated air temperature (heating) is often mentioned in the recent global climate change scenario. Species and cultivars of plants are characterized by a varying degree of sensitivity to the negative impact of the urban environment. Well-known effects include the accumulation of toxic compounds in cells, adverse changes in photosynthesis, respiration and transpiration intensity, loss of regulating capacity of the stomata, increase in leaf temperature, disturbance of synthesis intensity, and 
degradation of important metabolites (Lorenc-Plucińska and Byczyńska 1997). Furthermore, contaminants contribute to genetic changes, including those responsible for the photosynthesis process (Bahl et al. 1993). Roses can also react in different ways to abiotic stress conditions and simultaneously show various sensitivity depending on the taxon and the rootstock whether they are budded (Sharma et al. 2013).

Given that rose shrubs do not always grow on their own roots, but are often grafted, both the sensitivity of the noble cultivars and the rootstocks to the stress factors are important. New et al. (2008) found that most of the shrubs of three taxa tested as rootstock material [Rosa $\times$ fortuniana Lindl., $R$. multiflora Thunb., $R$. odorata (Andr.) Sweet] died after 15 weeks when watered with a solution of $\mathrm{NaCl} 9.0 \mathrm{dS} \cdot \mathrm{m}^{-1}$. The growth reduction in shrubs of all tested rootstocks was intensified as the concentration of saline solution increased, but the growth reduction in $R$. $\times$ fortuniana was smaller than in the other two rootstocks. Furthermore, the elevated levels of sodium and chlorine in water irrigation reduced in rootstocks relative chlorophyll concentration and the maximal photochemical efficiency of photosystem II (PSII). Rosa ×fortuniana appeared to be relatively more salinity tolerant and the $R$. multiflora was less (Niu et al. 2008). Other studies have shown that rootstocks were very different in this respect, e.g. $R$. ×noisettiana f. manetti (Crivelli ex Rivers) Rehder (syn. R. manetti Crivelli ex Rivers) and $R$. 'Natal Briar' were more resistant to salinity than $R$. odorata, $R$. multiflora, 'Rum 9', 'Dr. Huey' (Cabrera 2003). It was also indicated that $R$. multiflora and 'Dr. Huey', applied as rootstocks, reacted to the drought stress by plant growth reduction (Niu and Cabrera 2010).

Growth and development disturbances were found also in other rose cultivars than rootstocks. Rosa chinensis Jacq. responded to salinity stress and high temperature stress with no flowering, the induction of dormancy, leaf injury, leaf necrosis and chlorosis (Wahacome et al. 2001, Wang et al. 2009); $R$. rugosa Thunb. revealed a decrease of chlorophyll content, potential photochemical efficiency, performance index, and biomass accumulation when treated with deicing salt (Wrochna et al. 2010). Wilson (1981) found that Rosa sp. (considering quantity and quality) responded to light stress with a high light interception, increased photosynthesis rate, dry mass gain, ultimately flowering shoot quality improved in terms of a larger stem and bud diameter and increased fresh weight. Pécrix et al. (2011) reported that high temperature stress in Rosa sp. increased the gamete ploidy level and yellowing leaves. In studies of Hura et al. (2017) R. rubiginosa L. showed greater susceptibility to calcium chloride salinity than sodium chloride. Shrubs exhibited this as a greater dynamics of chlorosis, necrosis and leaf dryness, as well as the inhibition of photosynthetic activity. It also concerned changes in the anatomical structure of the palisade leaf crumb (Hura et al. 2017).

\section{ENVIRONMENTAL ASPECTS OF GROWING ROSES IN URBAN HABITATS}

The participation of roses in urban ecosystems is of high importance. The dense and thorny rose shrubs with nutritious hips are a safe place for birds and small 
mammals. They are used by (in brackets, the number of species) insects (100), birds (30), and mammals (27). Nectar and pollen attract the insects. Roses have entomogamous flowers that produce a lot of pollen to provide insects with valuable food. Rose flowers are visited by 10 species from the family of Cerambycidae, 10 from Curculionidae, 3 species of Hemiptera, 33 species of Tenthredinidae, 16 species of Aphidoidea, 31 species of smaller Lepidoptera and 10 species of wild bees (Popek 2002).

Popek (2002) indicated Rose species as suitable for open and park plantings, listing indigenous species, among others, R. gallica L., R. rubiginosa L., R. canina L. and domesticated $R$. rugosa Thunb., $R$. spinosissima L., and $R$. glauca Pourr. In addition, Popek (2002) indicated the above mentioned species as resistant to diseases, pests and air pollutants, especially fumes, and furthermore, he highlighted their low soil and nutrient requirements, thus being environmentally friendly. Planted on slopes, roses prevent the soil from wash-out (Popek 2002). They require research in terms of their phytoremediation capacity. The leaves of a shriveling rose have a small adsorption capacity of PM10 (micro-dust) and carbon dioxide, as well as limited adsorption of nitrogen oxides and ozone (Borowski et al. 2016, cit. Hoffman 2009).

Expanding the selection of historical roses, with a number of features suggesting their suitability for difficult growing conditions, contributes not only to the preservation of biodiversity of such cultivars and their genetic pools. High genetic variability within the species is a fundamental element of adaptation to diverse and unfavorable environmental conditions, including climate change. Environmental changes related to urbanization lead to the disturbance of numerous natural mechanisms functioning in ecosystems.

The impact of greenery on human well-being and health is also important. Drivers driving on green communication routes are less tired and more able to cope with stressful situations, which has an impact on reducing the number of accidents. In addition, formation of the road environment with the use of vegetation increases the pleasure of traveling of drivers and passengers (Szczepanowska 2008).

\section{ROSES FOR CULTIVATION IN URBAN CONDITIONS - PUBLIC AND HISTORIC GREENERY}

The possibility of growing roses, including the historical taxa, in urban conditions, draws the attention of many authors (Wizner 1970, Krüssmann 1974, Bugała 1991). Primarily, ground cover roses are suggested to be applied in the city (Borowski et al. 2016). Borowski et al. (2016) listed roses as suitable for many sites, not only connected to communication routes. The following roses taxa are recommended for planting in the most difficult conditions, including streets: $R$. canina, $R$. rugosa and $R$. ×rugotida 'Darthuis'. The latter two species produce suckers and therefore they are recommended for planting on slopes located in favorable growth conditions and near streets and highways (groups III and IV).

The selection of plants for sunny sites includes not only selected modern ground 
cover roses, but also several historical ones with a ground cover character, including 'Max Graf' (group V), R. rugosa 'Blanc Double de Coubert' (group IV), 'Hansa' (group IV) and 'Moje Hammarberg' (group IV).

For urban roadsides, the "Guidelines for Establishing and Maintaining Roadside Greens for the General Roads for National Roads and Motorways" list the rugosa rose as an "invasive species", which is not recommended (Maranda et al. 2013). However, this publication (2013) recommends two cultivars of hedgehog rose ( $R$. rugosa Thunb.), 'Moje Hammarberg' and 'Hansa', for urban areas, and the same are indicated in "Standards of shaping the greenery of Warsaw" (Borowski et al. 2016). Other park historical roses of the hybrid Rugosa group are similar to the above mentioned cultivars and able to act as the ground cover. These are 'Blanc Double de Coubert', 'Dagmar Hastrup', and 'George Will'. The cultivars 'Fimbriata', 'F.J. Grootendorst', and 'Pink Grootendorst' have flowers with frilled petals and they can be used also for hedges. These cultivars begin to bloom in the second to third decade of May, with repeat flowering and set decorative hips, except for the latter two.

\section{HISTORICAL ROSES OF HIGH POTENTIAL TO GROW IN URBAN CONDITIONS}

Numerous publications, as well as observations of growth and flowering in the climatic conditions of Central Poland in the Polish Academy of Sciences Botanical Garden in Powsin, Warsaw, indicate the great decorative value of many park rose cultivars (Monder 2007a, b, 2011, 2012a, 2014). They exhibit higher resistance to frost and winter hardiness than the recommended contemporary ground cover roses, which is a determinant of successful cultivation (Monder 2004, 2008, 2012c). Their decorative qualities, especially flowers and their fragrance, are high. In addition to the ornamental elements, many of them include purple or brown young shoots in spring, spiky shoots, hips, and autumn leaf discoloration. The low cultivation requirements allow to reduce the costs of the maintenance of green areas (Monder 2007a).

The French rose (Rosa gallica) and its cultivars are recognized as exceptionally resistant to frost and diseases, tolerant of semi-shadow sites, and well tolerating hot summers and harsh winters (Krüssmann 1974). The frost tolerance of this group reaches $30^{\circ} \mathrm{C}$ (Wizner 1970), which is confirmed by numerous observations in the Botanical Garden in Powsin (Monder 2014). They grow well even on dry, but quite fertile soils. The shrubs are usually compact, standing up, blooming on annual and older shoots. Some cultivars produce stolons. The hybrid Gallica group represents once-blooming cultivars with nice, scented flowers. The flowers are pink to dark-brown, usually very full and with a "green eye". Most of them are characterized by a dense growth and foliage, with outstanding resistance to frost and diseases. Numerous of them set ornamental hips, e.g., 'Complicata', 'Officinalis', 'Splendens', 'Violacea', 'Versicolor'. Other valuable cultivars in this group are, e.g., 'Ambroise Paré', 'Belle Herminie', 'Cardinal de Richelieu', 'Charles de Mills', 'Duchesse d'Angoulême', and 'Duchesse de Montebello' (Monder 2014) - the table. 


\begin{tabular}{|c|c|c|c|c|c|c|c|c|c|c|c|c|c|c|c|c|c|c|c|c|}
\hline $\begin{array}{l}\overrightarrow{\bar{\Xi}} \\
\bar{\Xi} \\
\overline{0}\end{array}$ & 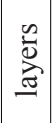 & 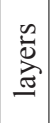 & 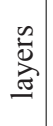 & & 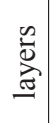 & 离 & & 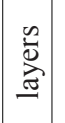 & & & & & & & & & & 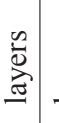 & 峁 & \\
\hline 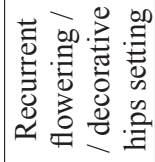 & $\stackrel{+}{+}$ & $\frac{+}{+}$ & $\stackrel{+}{+}$ & $\frac{1}{+}$ & & $\stackrel{+}{+}$ & $\frac{1}{1}$ & $\begin{array}{l}+ \\
+\end{array}$ & $\frac{1}{+}$ & $\frac{1}{+}$ & $\frac{1}{1}$ & $\frac{1}{1}$ & $\frac{1}{1}$ & $\stackrel{+}{+}$ & $\stackrel{+}{1}$ & $\frac{1}{1}$ & $\frac{1}{1}$ & + & + & \\
\hline 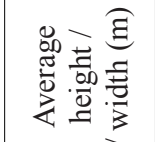 & 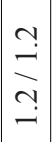 & 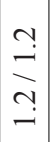 & $\begin{array}{c}\stackrel{\sim}{\Xi} \\
\stackrel{n}{N}\end{array}$ & $\stackrel{\sim}{\Xi}$ & 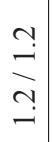 & $\begin{array}{l}n \\
\stackrel{n}{+} \\
-\end{array}$ & $\begin{array}{l}0 \\
i \\
0 \\
0 \\
0\end{array}$ & 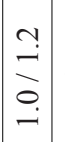 & 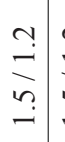 & 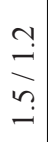 & $\frac{a}{0}$ & $\begin{array}{c}a \\
0 \\
\\
-1\end{array}$ & $\begin{array}{c}\hat{\sigma} \\
\dot{O} \\
\\
-\end{array}$ & \begin{tabular}{l|l}
$\stackrel{+}{N}$ & \\
$n$ & \\
- &
\end{tabular} & 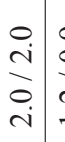 & 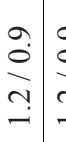 & 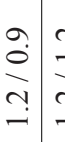 & 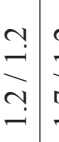 & 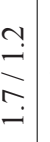 & 0 \\
\hline 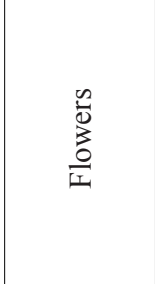 & 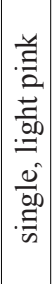 & 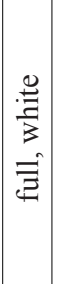 & 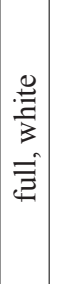 & 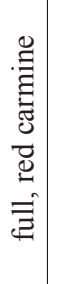 & 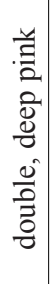 & 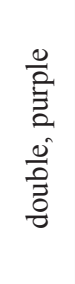 & 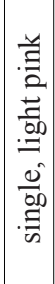 & 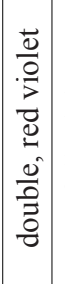 & 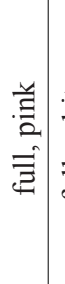 & 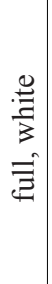 & 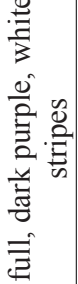 & 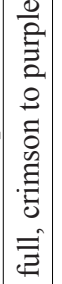 & $\mid$ & 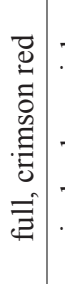 & 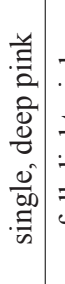 & 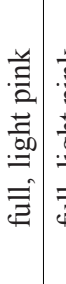 & 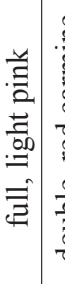 & 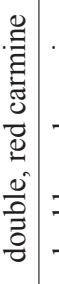 & 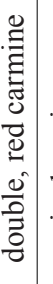 & \\
\hline $\begin{array}{l}\text { ?े } \\
\text { छే }\end{array}$ & $\begin{array}{l}\tilde{W} \\
2 \\
0 \\
\stackrel{0}{\approx} \\
\vec{\sim}\end{array}$ & $\mid \begin{array}{l}\mathscr{w} \\
0 \\
o \\
\vec{\alpha} \\
\vec{\alpha}\end{array}$ & $\mid \begin{array}{c}\mathscr{w} \\
0 \\
o \\
\overrightarrow{2} \\
\tilde{2}\end{array}$ & $\begin{array}{l}\tilde{\Xi} \\
0 \\
60 \\
\stackrel{\sim}{\simeq}\end{array}$ & $\begin{array}{l}\tilde{D} \\
0 \\
0 \\
\ddot{0} \\
\tilde{\sim}\end{array}$ & 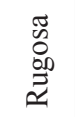 & 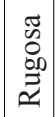 & 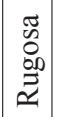 & 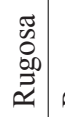 & $\begin{array}{c}\tilde{D} \\
\stackrel{0}{0} \\
\stackrel{0}{0} \\
\vec{\sim}\end{array}$ & : & 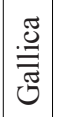 & 苞 & : & 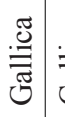 & $\stackrel{\mathscr{J}}{\stackrel{\Xi}{\widetilde{J}}}$ & 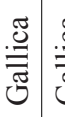 & 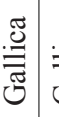 & . & 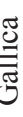 \\
\hline$\frac{.5}{0.00}$ & 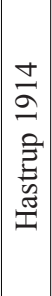 & 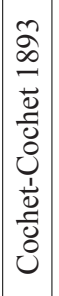 & 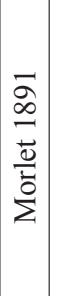 & $\begin{array}{l}\infty \\
\sigma \\
\vec{\sigma} \\
0 \\
0 \\
0 \\
0\end{array}$ & 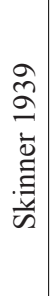 & 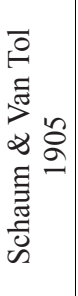 & 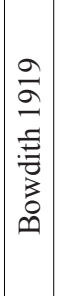 & 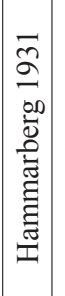 & 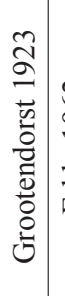 & 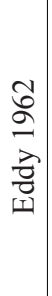 & \begin{tabular}{l}
0 \\
+ \\
$\infty$ \\
\multirow{1}{0}{} \\
0 \\
5
\end{tabular} & 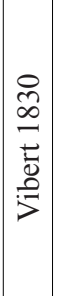 & 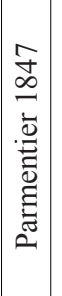 & 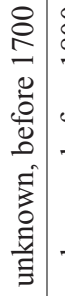 & 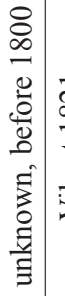 & 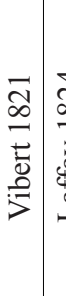 & 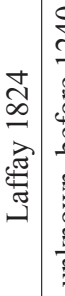 & 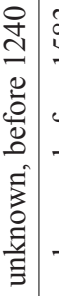 & 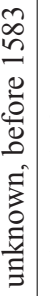 & ?2 \\
\hline 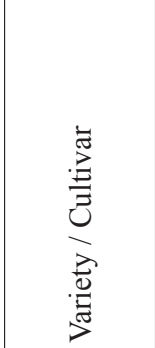 & 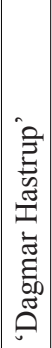 & 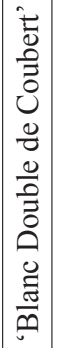 & 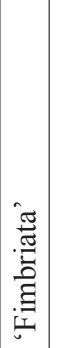 & 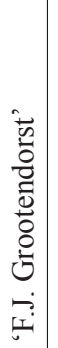 & $\mid \begin{array}{l}0 \\
3 \\
3 \\
0 \\
0 \\
0 \\
0 \\
0 \\
0\end{array}$ & 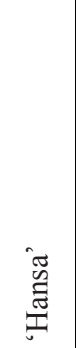 & 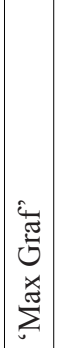 & 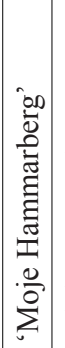 & 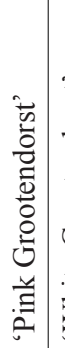 & 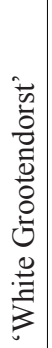 & 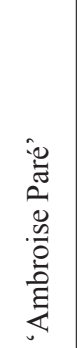 & 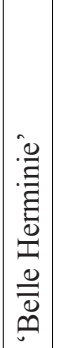 & 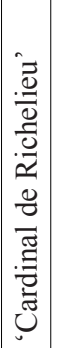 & 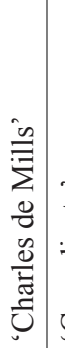 & 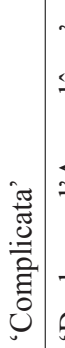 & $\begin{array}{c}0 \\
0 \\
0 \\
0 \\
0 \\
0 \\
0 \\
0 \\
0 \\
0 \\
0 \\
0 \\
0 \\
\overline{0} \\
\tilde{0}\end{array}$ & 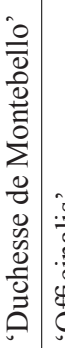 & 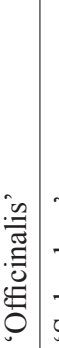 & 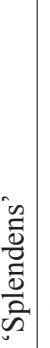 & \\
\hline
\end{tabular}




\begin{tabular}{|c|c|c|c|c|c|c|c|c|c|c|c|c|c|c|c|c|c|c|c|c|c|c|c|c|}
\hline 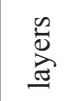 & 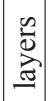 & & & & 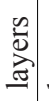 & 岕 & 岕 & 岕 & 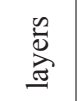 & & & & & & & & & & & & & & & \\
\hline$\stackrel{+}{i}$ & $\stackrel{+}{1}$ & $\frac{1}{1}$ & \pm & \pm & & & $\stackrel{+}{i}$ & $\frac{1}{+}$ & $\stackrel{+}{1}$ & $\frac{1}{1}$ & & \pm & $\frac{1}{1}$ & $\frac{1}{1}$ & $+\frac{+}{1}$ & & $\frac{1}{+}$ & $\frac{1}{+}$ & $\frac{1}{+}$ & $\frac{1}{+}$ & $\frac{1}{t}$ & $\frac{1}{+}$ & $\frac{1}{+}$ & $\frac{1}{+}$ \\
\hline 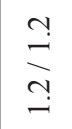 & 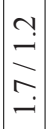 & $\begin{array}{l}\stackrel{0}{1} \\
i \\
0 \\
i\end{array}$ & $\begin{array}{l}0 \\
i \\
0 \\
i \\
i\end{array}$ & 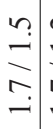 & 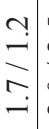 & $\begin{array}{l}n \\
i \\
o \\
o \\
i\end{array}$ & $\stackrel{\stackrel{N}{\Xi}}{\stackrel{N}{-}}$ & 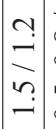 & $\begin{array}{ll}\infty & \infty \\
0 & 0 \\
0 & 0 \\
n & n \\
0 & 0\end{array}$ & 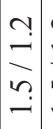 & 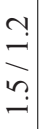 & $\begin{array}{l}n \\
i \\
n \\
i \\
i\end{array}$ & $\begin{array}{l}\stackrel{\sim}{\doteq} \\
\stackrel{0}{0} \\
i\end{array}$ & $\begin{array}{l}\stackrel{o}{i} \\
\dot{i} \\
\dot{i} \\
i\end{array}$ & 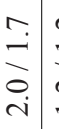 & 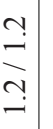 & 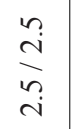 & \begin{tabular}{c|}
$\infty$ \\
0 \\
0 \\
0 \\
$\dot{-}$
\end{tabular} & $\mid \begin{array}{c}\infty \\
0 \\
0 \\
\\
-1\end{array}$ & $\begin{array}{l}\stackrel{\infty}{0} \\
\stackrel{0}{0}\end{array}$ & & $\frac{a}{\tilde{O}}$ & 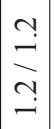 & 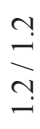 \\
\hline 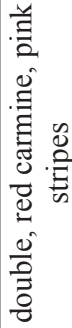 & 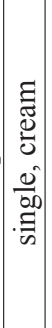 & 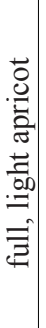 & $\begin{array}{l}3 \\
0 \\
0 \\
0 \\
2 \\
0 \\
0 \\
0 \\
0 \\
0 \\
.0 \\
. \frac{1}{0} \\
0 \\
0 \\
0\end{array}$ & 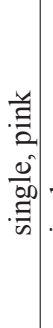 & 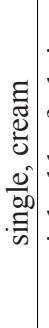 & 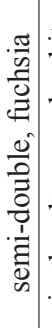 & 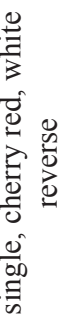 & 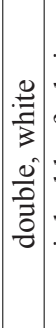 & 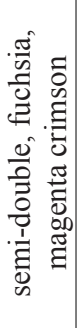 & 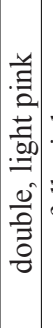 & 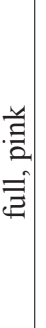 & 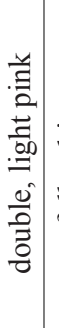 & 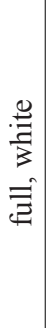 & 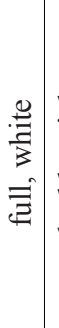 & 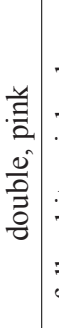 & 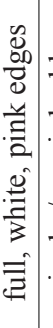 & 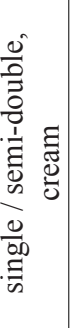 & 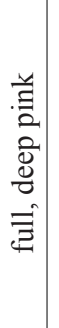 & 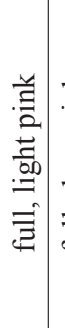 & 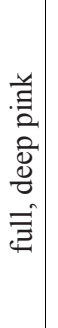 & 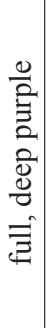 & 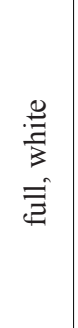 & 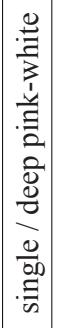 & 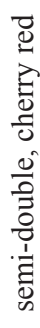 \\
\hline : & 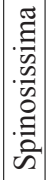 & 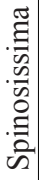 & 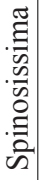 & 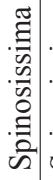 & 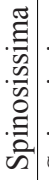 & 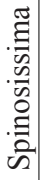 & 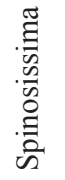 & 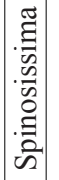 & 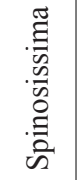 & 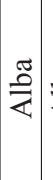 & 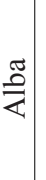 & $\begin{array}{l}\tilde{0} \\
\stackrel{2}{\psi}\end{array}$ & $\stackrel{\pi}{=}$ & $\stackrel{\pi}{ٍ}$ & 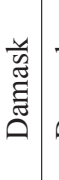 & 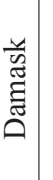 & 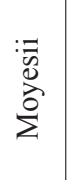 & 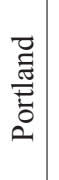 & 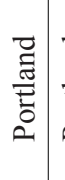 & 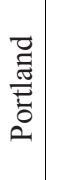 & $\stackrel{\frac{\pi}{2}}{\stackrel{0}{\oplus}}$ & 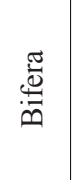 & $\frac{y}{\tilde{s}}$ & $\frac{y}{s}$ \\
\hline 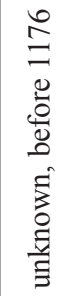 & $\begin{array}{c}\text { 咅 } \\
\text { 亲 } \\
\text { 豙 }\end{array}$ & 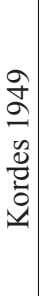 & 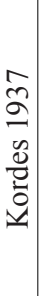 & 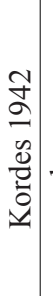 & 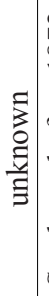 & 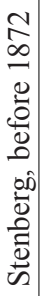 & 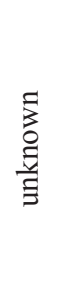 & $\begin{array}{l}\tilde{\Xi} \\
\vdots \\
\vdots \\
\vdots \\
\Xi\end{array}$ & 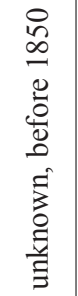 & 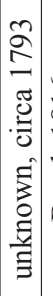 & $\begin{array}{l}0 \\
0 \\
\infty \\
\vec{c} \\
\underline{0} \\
0 \\
\infty\end{array}$ & $\begin{array}{l}\infty \\
2 \\
\infty \\
- \\
\tilde{E} \\
\vdots \\
0 \\
\vdots \\
\vdots \\
\vdots\end{array}$ & 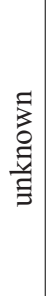 & 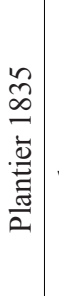 & $\begin{array}{l}\frac{\Xi}{3} \\
0 \\
\vdots \\
\Xi\end{array}$ & $\begin{array}{l}\text { 志 } \\
0 \\
\vdots \\
\text { 音 }\end{array}$ & 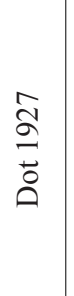 & 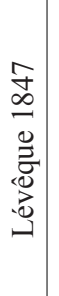 & 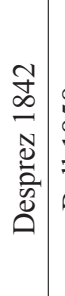 & $\begin{array}{l}\infty \\
\infty \\
\infty \\
= \\
\overline{0} \\
\infty\end{array}$ & 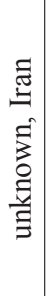 & 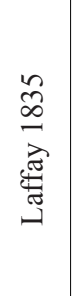 & 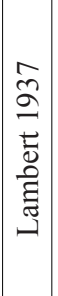 & 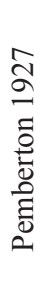 \\
\hline 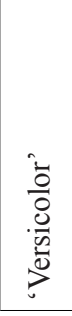 & 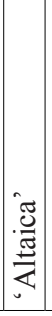 & 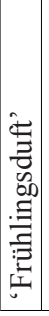 & & 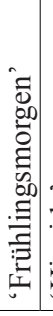 & 을 & 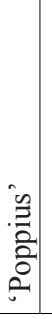 & $\begin{array}{l}\vec{D} \\
0 \\
0 \\
0 \\
0 \\
: 0 \\
0\end{array}$ & 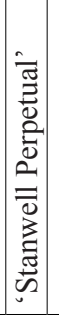 & 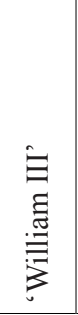 & 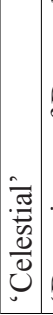 & 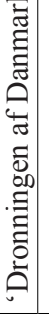 & 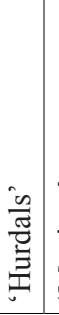 & 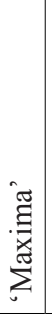 & 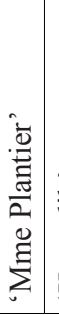 & 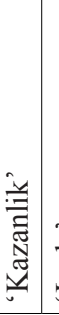 & . & 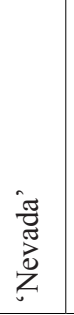 & 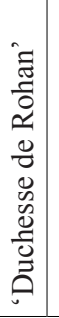 & 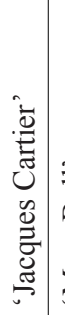 & $\begin{array}{l}\overline{\overline{0}} \\
\overline{0} \\
\bar{g} \\
\sum_{0}\end{array}$ & $\begin{array}{l}\bar{z} \\
0 \\
0 \\
0 \\
\simeq \\
0 \\
0 \\
0 \\
0 \\
0 \\
0 \\
0\end{array}$ & 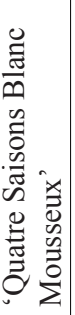 & 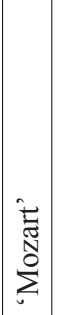 & 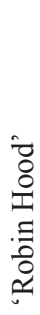 \\
\hline
\end{tabular}


Historical roses from the Rugosa group do not differ from modern cultivars (Monder 2012b). Among many cultivars, 'F.J. Grootendorst' and its sport 'Pink Grootendorst' are resistant to frost, and repeat their flowering, producing interesting flowers resembling miniature cloves (Monder 2012a), likewise 'White Grootendorst'. Observations made by Monder (2012a) confirm high decorative values, frost and disease resistance of the following repeat blooming cultivars: 'Blanc Double de Coubert', 'Frau Dagmar Hastrup', 'Hansa', 'Moje Hammarberg'. They form decorative hips, and their leaves resemble those of the species and turn yellow in autumn. These latter four cultivars create stolons, which makes them suitable, for example, for the consolidation of slopes. The height of the shrubs of particular cultivars varies from 0.5 to $2.0 \mathrm{~m}$; they are dense, and perfect for hedges, while lower ones for cover (the table).

The Pimpinellifolia hybrids are very popular in the Nordic countries of Europe and they are often planted in public green areas, parking lots and along the streets and communications routes. The cultivars of Pimpinellifolia hybrids often bloom at the end of the second decade of May and then set decorative black-purple hips. The cultivars of older origin can also be recommended, for example: 'Altaica', 'Frühlingsduft', 'Frühlingsgold', 'Frühlingsmorgen', 'Hispida', 'Poppius', 'Single Cherry', 'Stanwell Perpetual', 'William III' (the table). Most cultivars of this group are very resistant to frost and their leaves turn yellow or red in autumn (Monder 2011). Pimpinellifolia hybrids have low soil requirements, tolerate semi-shaded sites, and bloom on at least the previous-year's shoots. They need minor care, which is similar to the Rugosa hybrids. In wet years, some cultivars can be infected by black spots.

The Alba roses include cultivars derived from Rosa $\times$ alba L. Shrubs are usually wide, high $(1.53 .0 \mathrm{~m})$, loosely built, and their white flowers are shady pink and fragrant. Grey-green foliage, sometimes blue shoots, and very few prickles, are characteristic features. They tolerate both sunny and slightly shaded sites. Shrubs usually grow healthy (in some areas they may be affected by rust), and are very resistant to frost. Valuable cultivars include 'Hurdals', 'Dronningen af Danmark', 'Celestial', 'Maxima', and 'Mme Plantier' (Monder 2007a, b) - the table.

The Damask roses are not very high shrubs (1.0-1.7 m), with stems having characteristic reddish prickles and stiff bristles, and a loose conformation, sometimes with stolons and leaves bent slightly down. The flowers are pink, rarely white, usually semi-full or very full, with a "green eye". The petals of some cultivars are used to produce rose oil. They tolerate weaker and drier soils. The frost resistance of most of these cultivars is high ('Leda'), though, rarely, younger, one-year shoots are partially frozen in cold winters ('Kazanlik') (Monder $2007 a, b)$ - the table.

Other park roses not belonging to so numerous groups are also worth mentioning. They include the early-blooming (second decade of May) $R$. xanthina Lindl., the great attraction of which is its abundant yellow flowers, then dark shoots and fragile leaves. It is able to withstand frost up to $25^{\circ} \mathrm{C}$ (Wizner 1970). Cultivars of $R$. moyesii, 'Nevada' (Monder 
2007a), and its sport 1959, 'Marguerite Hilling', blooming at the end of May, are also valuable. The shrubs are high (over $2 \mathrm{~m}$ ) with attractive large flowers and a weeping conformation (the table). In humid summers, some Moyesii hybrids can be infected by black spots.

Among the repeat blooming cultivars, especially suitable for presentable places in historical gardens, there are those of the Portland and Bifera groups. The Portland 'Duchesse de Rohan', 'Jacques Cartier', and 'Mme Boll' and the Bifera 'Rose de Rescht', and 'Quatre Saisons Blanc Mousseux' can be recommended. They are characterized by their compact growth, and their height does not usually exceed $1.2 \mathrm{~m}$. Musk hybrids are also mentioned as repeat-blooming cultivars. The flowers are small, in shades of pink, fragrant, gathered in large inflorescences on the shoot tops. The shrubs are very dense, spherical in shape, 1 to $2 \mathrm{~m}$ high. Their frost resistance is usually high, but in harsh winters, the shrubs may suffer. However, after pruning they regenerate and bloom well in the same year. Valuable cultivars are, e.g. 'Mozart' and 'Robin Hood' (Monder 2007a) - the table.

\section{CONCLUSIONS}

In Poland, there is no tradition of the application of historical roses in public green areas, which is in contrast to other countries with gardening of a high culture. However, some of the historical cultivars can be recommended for planting along transport routes and in urban green areas, especially in the public greenery, communal gardens, parks and in all places of historical importance.
Many of them can be used in naturalistic planting schemes for garden landscapes. Roses can be planted solitary, in groups, for mass planting, as hedges, in sunny or partially shaded places. They are also ideal for compositions including other species by arranging plants in loose mingled combinations. The possibilities of using the park roses are similar to other deciduous shrubs. It is worth emphasizing that these roses usually bloom early, from the 3rd decade of May, i.e. before the cultivars of most of the ground cover, floribunda and other groups of roses - so they can prolong blooming in the garden, especially of that of the one leading theme which is roses. The charm and scent of rose flowers at this time of the year is complemented by late spring and early summer compositions. A good possibility for the use of historical park roses is the revitalization of urban parks, representative spaces and green areas, where the direct contact of humans with plants is greater than in the city's communication space. They should find a permanent place in different types of historical gardens.

Additional aspects of their use are social and ecological values. Greenery has a positive effect on the well-being of people, as well as it reduces the tension and stress associated with transport.

\section{REFERENCES}

BACH A., FRAZIK-ADAMCZYK M. 2006: Charakterystyka zagrożeń zieleni miejskiej ze szczególnym uwzględnieniem zieleni w ciagach komunikacyjnych. Opracowanie wykonane dla Urzędu Miasta Krakowa. Wydział Gospodarki Komunalnej i Ochrony Środowiska, Kraków. 
BARANOWSKI T. 2010: Wpływ zabiegów pielęgnacyjnych na zdrowotność drzew w miastach. Prog. Plant Prot. 50 (4): 1569-1574.

BOROWSKI J., FORTUNA-ANTOSZKIEWICZ B., ŁUKASZKIEWICZ J., ROSŁON-SZERYŃSKA E., SITARSKI M., SUCHOCKA M., WYSOCKI C. 2016: Standardy kształtowania zieleni Warszawy [Standards of shaping the greenery of Warsaw]. Załącznik do Ustawy z 15.12.2016 r. Rady m.st. Warszawy.

BUGAŁA W. 1991: Drzewa i krzewy dla terenów zieleni. PWRiL, Warszawa.

CABRERA R.I. 2003: Demarcating salinity tolerance in greenhouse roses. Acta Hort. 609: 51-57.

CAIRNS T. 2003: Classification / Horticultural classification schemes. In: A.V. Roberts, T. Debener, S. Gudin (Eds.) Encyclopedia of Rose Science. Vol. 1. Elsevier, Oxford: 117-124.

HURA T., SZEWCZYK-TARANEK B., HURA K., NOWAK K. 2017: Physiological responses of Rosa rubiginosa to saline environment. Water Air Soil Pollut. 228: \# 81. doi: 10.1007/s11270-0173263-2

KRÜSSMANN G. 1974: Rosen Rosen Rosen. Unser Wissen über die Rosen. Paul Parey, Berlin - Hamburg.

LORENC-PLUCIŃSKA G., BYCZYŃSKA A. 1997: Reakcje roślin na spaliny samochodowe. In: International Scientific Meeting Ecophysiological aspects of plants responses to stress factors. Kraków, 12-14.06.1997: 41-49.

MARANDA K., KARPOWICZ P., KOSEK M., KUCHARSKA M., MATEREK T., MLECZKO-KRÓL M., MUSIEL M., OCHNIO P., SIEDLECKI T., STANKIEWICZ E., WÓJCIKOWSKA I. 2013: Wytyczne zakładania i utrzymania zieleni przydrożnej na potrzeby Generalnej Dyrekcji Dróg Krajowych i Autostrad [Guidelines for Establishing and Maintaining Roadside Greens for the General Roads for National Roads and Motor- ways]. Generalna Dyrekcja Dróg Krajowych i Autostrad, Warszwa.

MĘDRZYCKI P. 2007: Zmniejszanie śmiertelności młodych drzew w mieście. Przegląd Komunalny 8: 65-67.

MONDER M.J. 2004: Observations of frost resistance of cover roses in the roses collection in the Botanical Garden of the Polish Academy of the Sciences in Warsaw after frosty winter 2002/2003. Bulletin of Botanical Gardens. Museums \& Collections 13: 187-198.

MONDER M.J. 2007a: Charakterystyka wzrostu i wartości dekoracyjnej wybranych odmian parkowych róż historycznych. In: XI Ogólnopolska Konferencja Szkółkarska Problemy i perspektywy produkcji szkółkarskiej roślin ozdobnych. Skierniewice, 20-21.02.2007: 31-40.

MONDER M.J. 2007b: Zasoby genowe i ocena wybranych odmian róż historycznych w kolekcji Ogrodu Botanicznego CZRB PAN. ZPPNR 517 (2): 487-494.

MONDER M.J. 2008: Ocena wzrostu i kwitnienia 10 wybranych odmian róż z grupy okrywowych. ZPPNR 25: 261-269.

MONDER M.J. 2011: Evaluation of growth and flowering of cultivars derived from the Pimpinellifolia (Rosa pimpinellifolia L.) growing in the collection of rose cultivars in the Botanical Garden of the Polish Academy of Sciences in Powsin. J. Fruit Ornam. Plant Res. 19 (1): 195-207.

MONDER M.J. 2012a: Evaluation of growth and flowering of cultivars derived from the Rugosa (Rosa rugosa Thunb.) growing in the National Collection of Rose Cultivars in the Polish Academy of Sciences Botanical Garden in Powsin. Part I. The historical cultivars. Acta Agrobot. 65 (2): 109-116.

MONDER M.J. 2012b: Evaluation of growth and flowering of cultivars derived from the Rugosa (Rosa rugosa Thunb.) growing in the National Collection of Rose Cultivars in the Polish Academy of Sciences Botanical Garden in Powsin. Part II. The modern cultivars. Acta Agrobot. 65 (2): 107-124. 
MONDER M.J. 2012c: Evaluation of Growth and Flowering of 15 Modern Ground Cover Cultivars of Roses Growing in the Collection of Rose Cultivars in the Polish Academy of Science's Botanical Garden in Powsin. Acta Hortic 953: 8590.

MONDER M.J. 2014: Evaluation of growth and flowering of historical cultivars of the Rosa gallica L. growing in the National Collection of Rose Cultivars in the Polish Academy of Science Botanical Garden in Powsin. Acta Agrobot. 67: 39-52.

NIU G., RODRIGUEZ D.S., AGUINIGA L. 2008: Effect of saline water irrigation on growth and physiological responses of three rose rootstocks. HortSci 43 (5): \# 1479 .

NIU G., CABRERA R.I. 2010: Growth and physiological responses of landscape plants to Saline Water Irrigation: A Review. HortSci 45 (11): 1605-1609.

Generalna Dyrekcja Ochrony Środowiska 2016: Ogrodnictwo wobec roślin inwazyjnych obcego pochodzenia - kodeks dobrych praktyk [Horticulture against invasive plants of foreign origin a code of good practice]. Warszawa. Retrieved from: http://www.gdos.gov. pl/files/aktualnosci/31085/Kodeks_Dobrych_Praktyk_Ogrodnictwo_wo bec roslin_inwazyjnych_obcego_pochodzenia_www_2016_08_12_news_image. pdf.

PÉCRIX Y., RALLO G., FOLZER H., CIGNA M., GUBIN S., Le BRIS M. 2011: Polyploidization mechanisms: temperature environment can induce diploid gamete formation in Rosa sp. J. Exp. Bot. 62 (10): 3587-3597.

POPEK R. 2002: Róże dziko rosnące Polski. Klucz - atlas. Plantpress, Kraków.

SHARMA M., KAUL K., KAUR N., SINGH M., DHAYANI D., AHUJA P.S. 2013: Rose: Improvement for Crop Productivity. In: N. Tuteja, S.S. Gill, R. Tuteja (Eds.) Improving Crop Productivity in Sustainable Agriculture. Wiley-VCH: 485-506.
SZCZEPANOWSKA H.B. 2008: Drzewa w otoczeniu ulic - problemy inżynieryjne, społeczne, ekonomiczne oraz bezpieczeństwa. Człowiek i Środowisko 35: 19-34.

WAHOME P.K., JESCH H.H., PINKER I. 2001: Effect of sodium chloride stress, decreases malondialdehyde content and affects petal color of salt stressed cut rose (Rosa ×hybrida L.) 'Hot Lady'. Afr. J. Biotechnol. 8: 1502-1508.

WANG T., TONG Z., MA N., GAO J. 2009: Isolation and expression analysis of Rh-DREB1s gene in cut roses (Rosa hybrida) under ethylene treatment and water deficit stress. Acta Hortic. Sinica 36 (1): 65-72.

WILSON J.W. 1981: Analysis of light interception by single plants. Ann. Bot. 48: 501-505.

WIZNER K. 1970: Róże w ogródku. PWRiL, Warszawa.

WROCHNA M., MALECKA-PRZYBYSZ M., GAWROŃSKA H. 2010: Effect of road de-icing salt with anti corrosion agents on selected plant species. Acta Sci. Pol. Hortorum Cultus 9 (4): 171-182.

Streszczenie: Parkowe róże historyczne w przestrzeni miejskiej. Tradycja uprawy róż sięga czasów antycznych i do dziś są one jedną z najważniejszych roślin ozdobnych. Liczne gatunki i odmiany starszego pochodzenia mają duże walory dekoracyjne, a ponadto spełniają warunki stawiane roślinom przeznaczonym do sadzenia w mieście: są bardzo odporne na warunki zimowe i rosną dobrze na własnych korzeniach; tolerują upały oraz susze; są wystarczająco odporne na choroby i szkodniki; mają zróżnicowane i nieduże wymagania glebowe; „,czysto” przekwitaja; maja niewielkie wymagania pielęgnacyjne sprzyjające ograniczeniu nakładów finansowych. Istotny jest ich udział w ekosystemach miejskich: gęste i kolczaste krzewy róż stanowią schronienie, a pseudoowoce pożywienie dla ptaków i małych ssaków; nektar i pyłek kwiatowy wabią owady. W Polsce nie ma tradycji stosowania róż historycznych w zieleni publicznej. Możliwy odpowiedni dobór róż historycznych o wymienionych korzyst- 
nych cechach dotyczy terenów zurbanizowanych, w tym na przydroża, do zieleńców i parków, a przede wszystkim do założeń historycznych i zabytkowych. Wiele róż z tej grupy jest zalecanych do założeń o charakterze naturalistycznym. Wśród nich są taksony odpowiednie do stosowania w nasadzeń rabatowych, grupowych, jako żywopłoty nieformowane, do kompozycji z innymi gatunkami roślin na stanowiskach słonecznych lub częściowo zacienionych.

Stowa kluczowe: krajobraz miejski, ogrodnictwo miejskie, rozarium, róże historyczne, park, ogród historyczny
MS received: 20.09 .2017

MS accepted: 16.05.2018

Author's address:

Marta Joanna Monder

Polska Akademia Nauk Ogród Botaniczny

- Centrum Zachowania Różnorodności

Biologicznej

ul. Prawdziwka 2, 02-973 Warszawa

Poland

e-mail: mondermarta@obpan.pl 